OPEN ACCESS

Edited by:

Nicolas Rouhier,

Université de Lorraine, France

Reviewed by:

Carlos García-Mata

National University of Mar del Plata,

Argentina

Olivier Keech,

Umeå University, Sweden

*Correspondence:

Guangdong Yang

gyang2@laurentian.ca

Yanxi Pei

peiyanxi@sxu.edu.cn

Specialty section:

This article was submitted to

Plant Physiology,

a section of the journal

Frontiers in Plant Science

Received: 05 June 2018 Accepted: 06 November 2018

Published: 23 November 2018

Citation:

Jin Z, Sun L, Yang G and Pei Y (2018) Hydrogen Sulfide Regulates Energy Production to Delay Leaf Senescence Induced by Drought

Stress in Arabidopsis.

Front. Plant Sci. 9:1722.

doi: 10.3389/fp/s.2018.01722

\section{Hydrogen Sulfide Regulates Energy Production to Delay Leaf Senescence Induced by Drought Stress in Arabidopsis}

\author{
Zhuping Jin ${ }^{1}$, Limin Sun ${ }^{1}$, Guangdong Yang ${ }^{1,2 *}$ and Yanxi Pei ${ }^{1 *}$ \\ ${ }^{1}$ School of Life Science, Shanxi University, Taiyuan, China, ${ }^{2}$ Department of Chemistry and Biochemistry, Laurentian \\ University, Sudbury, ON, Canada
}

Hydrogen sulfide $\left(\mathrm{H}_{2} \mathrm{~S}\right)$ is a novel gasotransmitter in both mammals and plants. $\mathrm{H}_{2} \mathrm{~S}$ plays important roles in various plant developmental processes and stress responses. Leaf senescence is the last developmental stage and is a sequential degradation process that eventually leads to leaf death. A mutation of the $\mathrm{H}_{2} \mathrm{~S}$-producing enzymeencoding gene L-cysteine desulfhydrase1 (DES1) leads to premature leaf senescence but the underlying mechanisms are not clear. In this present study, wild-type, DES1 defective mutant (des1) and over-expression (OE-DES1) Arabidopsis plants were used to investigate the underlying mechanism of $\mathrm{H}_{2} \mathrm{~S}$ signaling in energy production and leaf senescence under drought stress. The des 1 mutant was more sensitive to drought stress and displayed accelerated leaf senescence, while the leaves of OE-DES1 contained adequate chlorophyll levels, accompanied by significantly increased drought resistance. Under drought stress, the expression levels of ATP $\beta-1,-2$, and -3 were significantly downregulated in des 1 and significantly upregulated in OE-DES1, and ATPE showed the opposite trend. Senescence-associated gene (SAG) 12 correlated with agedependent senescence and participated in the drought resistance of OE-DES1. SAG13, which was induced by environmental factors, responded positively to drought stress in des 1 plants, while there was no significant difference in the SAG29 expression between des 1 and OE-DES1. Using transmission electron microscopy, the mitochondria of des 1 were severely damaged and bubbled in older leaves, while OE-DES1 had complete mitochondrial structures and a homogeneous matrix. Additionally, mitochondria isolated from OE-DES1 increased the $\mathrm{H}_{2} \mathrm{~S}$ production rate, $\mathrm{H}_{2} \mathrm{~S}$ content and ATPase activity level, as well as reduced swelling and lowered the ATP content in contrast with wildtype and des 1 significantly. Therefore, at subcellular levels, $\mathrm{H}_{2} \mathrm{~S}$ appeared to determine the ability of mitochondria to regulate energy production and protect against cellular aging, which subsequently delayed leaf senescence under drought-stress conditions in plants.

Keywords: hydrogen sulfide, mitochondrion, ATPase activity, energy production, leaf senescence, drought stress

Abbreviations: ADP, adenosine diphosphate; ATP, adenosine triphosphate; CYS, cyanoalanine synthase; Cys, cysteine; DES1, cysteine desulfhydrase; $F_{0} / F_{1}$, complex of ATP synthase; $\mathrm{H}_{2} \mathrm{~S}$, hydrogen sulfide; LCD/DCD, L/D-cysteine desulfhydrase; NFS1, cysteine desulfurase; OASTL, O-acetyl-L-serine(thiol)lyase; SAGs, senescence associated genes; TCA, tricarboxylic acid. 


\section{INTRODUCTION}

Hydrogen sulfide $\left(\mathrm{H}_{2} \mathrm{~S}\right)$ is an important member of the gasotransmitter family, along with nitric oxide and carbon monoxide, in both mammals and plants (Wang, 2012). $\mathrm{H}_{2} \mathrm{~S}$ has a wide range of physiological roles that are critical for plant development, such as seed germination (Zhang et al., 2008), root elongation (Zhang et al., 2009), flowering (Zhang et al., 2011), leaf senescence (Álvarez et al., 2010) and fruit maturation (Dooley et al., 2013). $\mathrm{H}_{2} \mathrm{~S}$ not only regulates the progress of cell viability (Álvarez et al., 2010), autophagy (Álvarez et al., 2012; Gotor et al., 2013), stomatal movement (Jin and Pei, 2016) and photosynthesis (Chen et al., 2011), but also mediates tolerance and protection against many different environmental stresses, including heat ( $\mathrm{Li}$ et al., 2012), cold (Du et al., 2017), drought (Jin et al., 2011, 2013, 2017), heavy metals (Fang et al., 2016, 2017), and others (Jin and Pei, 2015). $\mathrm{H}_{2} \mathrm{~S}$ is endogenously produced and metabolized in a precise and regulated manner. Cysteine degradation by CDes catalyzes the formation of sulfide, ammonia and pyruvate in a 1:1:1 stoichiometric ratio (Papenbrock et al., 2007). Plant cells contain different CDes localized in the cytoplasm, plastids and mitochondria (Jin and Pei, 2015). One class of these enzymes, L-Cys desulfhydrases, containing LCD (At3g62130) and DES1 (At5g28030), localize in the nucleus and cytoplasm, respectively. DES1 is regarded as the major contributor to the generation of $\mathrm{H}_{2} \mathrm{~S}$ (Álvarez et al., 2010).

Leaf senescence, as the last developmental stage, occurs gradually and is characterized by specific macroscopic, cellular, biochemical and molecular changes. Nutrient reallocation is accompanied by organelle breakdown, energy reduction and gene expression during senescence (Munné-Bosch and Alegre, 2004). Mitochondria, the energy powerhouses, play important roles in the cell redox homeostasis, and mitochondrial ATP production is required in both heterotrophic and photosynthetic cells (Noctor et al., 2007). The conversion of the electrochemical proton gradient into ATP is catalyzed by an ATP synthase, often called $\mathrm{F}_{0} \mathrm{~F}_{1}$-ATPase, which is located in the inner mitochondrial membrane (Rondelez et al., 2005). ATP synthesis occurs sequentially on the three $\beta$-subunits of the $\mathrm{F}_{1}$-ATPase $(\alpha 3 \beta 3 \gamma \delta \varepsilon)$, while the $\varepsilon$ subunit is an endogenous inhibitor of the ATPase activity of $F_{1}$ (Kato et al., 1997). The decreased activity of the $\mathrm{F}_{1}$-ATPase may be the main cause of age-dependent mitochondrial dysfunction (Kadoya et al., 2011). Age-related deficits and damage to cellular macromolecules involved in energy production may underlie the age-related lowered ATP production level (Wang et al., 2003). ATP levels drop significantly in stressed cells and increase in the recovered cells (Sõti et al., 2003). Senescence-associated gene (SAG) 12,13 and 29 are widely used as molecular markers for leaf senescence (Chen et al., 2014).

Mitochondrial morphology and function are preserved by $\mathrm{H}_{2} \mathrm{~S}$ when mammals suffer sepsis (Aslami et al., 2013). The endogenous $\mathrm{H}_{2} \mathrm{~S}$ remains a regulator of energy production in mammalian cells under stress conditions (Fu et al., 2012) and delays cellular senescence by attenuating oxidative stress (Yang et al., 2013). In Arabidopsis thaliana, a mutation of the DES1 gene leads to early-flowering, premature leaf senescence and cadmium sensitivity (Álvarez et al., 2010). The mRNA levels of $\mathrm{H}_{2} \mathrm{~S}$-encoding genes, $\mathrm{L} / \mathrm{D}$-CDes, are gradually elevated in a developmental stage-dependent manner, and the $\mathrm{H}_{2} \mathrm{~S}$ production rate is positively correlated with the extent of the drought stress (Jin et al., 2011). $\mathrm{H}_{2} \mathrm{~S}$ prolongs the longevity of fresh-cut flowers and kiwifruit, suggesting that the role of $\mathrm{H}_{2} \mathrm{~S}$ might be universal in plant senescence (Zhang et al., 2011; Gao et al., 2013). However, the underlying mechanisms of $\mathrm{H}_{2} \mathrm{~S}$ signaling in plant senescence and the aging process remain unclear.

Plant aging and environmental stresses may induce the process of senescence. Drought induces several responses in plants, including leaf senescence, which plays a major role in the survival of several species (Munné-Bosch and Alegre, 2004). In the present study, we focused on the role of endogenous $\mathrm{H}_{2} \mathrm{~S}$ signals as responses for plant survival and, more specifically, on how these signals contribute to the energy production of mitochondria during leaf senescence under drought-stress conditions.

\section{MATERIALS AND METHODS}

\section{Plant Materials and Growth Conditions}

Arabidopsis thaliana ecotype Columbia-0 (wild-type, WT) and T-DNA insertion mutant of DES1 (SALK_205358C) seeds were obtained from the Arabidopsis Biological Resource Center $\left(A B R C^{1}\right)$. The result of genotyping showed that des1 was a null mutant (Supplementary Figure S1). Construction of a transformation vector (35S::DES1) and transformation of Columbia- 0 by the floral dip method were performed previously. Transformants were selected on 1/2 MS agar plates containing $20 \mathrm{mg} \mathrm{L}^{-1}$ hygromycin. The T3 seeds which did not segregate on $1 / 2$ MS plates with hygromycin, were identified as homozygous OE lines. After the comparison of seed germination under PEG8000 stress and qRT-PCR analysis, one of them was used in the following experiments (Supplementary Figure S2). Seeds of WT, homozygous transgenic (OE-DES1) and mutant (des1) were vernalized for $2 \sim 4$ days at $4^{\circ} \mathrm{C}$ and then grown in a nutrient soil: vermiculite $(1: 1, \mathrm{v} / \mathrm{v})$ mixture in a growth chamber. The growth chamber was maintained at $23 \pm 1^{\circ} \mathrm{C}$ with $60 \%$ relative humidity, light illumination of $160 \mu \mathrm{E} \cdot \mathrm{m}^{-2} \cdot \mathrm{s}^{-1}$ and a photoperiod of 16/8 h (light/dark).

The 4-week-old WT, des1 and OE-DES1 seedlings were subjected to drought stress by withholding water for 7 days, and their growth states were recorded. Each associated gene expression level was determined in WT, des1 and OE-DES1. The leaves of plants were collected, immediately frozen in liquid nitrogen and stored at $-80^{\circ} \mathrm{C}$.

\section{Extraction of Total RNA and Quantitative Real-Time PCR (qRT-PCR)}

Total RNA was extracted using TRIzol ${ }^{\circledR}$ Reagent (Invitrogen) according to the manufacturer's instructions. Then, the RNA sample, M-MLV reverse transcriptase and an oligo(dT18) primer (TransGen Biotech) were used to synthesize complementary cDNA, and qRT-PCR was performed using a Bio-Rad real-time

${ }^{1}$ http://www.arabidopsis.org/abrc/ 
PCR Detection System (in a CFX96TM C1000 thermal cycler). In the relative quantification analysis, the gene $U B Q 4$ was used as the internal control. Each experiment was performed in triplicate and repeated independently with three biological replicates (Table 1).

\section{Observation of Mitochondrial Ultrastructure Using Transmission Electron Microscopy (TEM)}

To analyze the ultrastructure of mitochondria by TEM, 2-, 4-, 6-, and 8-week-old plants were subjected to drought stress and cultured in pots without water for 1 week. Leaves were collected and fixed for $24 \mathrm{~h}$ in $2.5 \%$ glutaraldehyde in $0.1 \mathrm{M}$ phosphate buffer $(\mathrm{pH} 7.2)$ at $4^{\circ} \mathrm{C}$, and then thoroughly washed with the same buffer three times ( $10 \mathrm{~min}$ per wash). Subsequently, samples were post-fixed in $2 \%$ osmium tetroxide in the same buffer for $4 \mathrm{~h}$. Samples were dehydrated gradually by increasing concentrations of ethyl alcohol $(25,50,75,85,95$, and $100 \%)$ and embedded in Epon 812 resin. Following overnight embedding and the polymerization of the embedding medium, ultrathin sections were cut using an ultramicrotome (LACA-UC6) with a diamond knife $(70 \mathrm{~nm})$. Finally, samples were stained with uranyl acetate and lead citrate. They were then examined using a transmission electron microscope (JEOL, JEM-1400) and photographed.

\section{Isolation and Purification of Mitochondria From Leaves}

The intact mitochondria were isolated and purified following a protocol described previously, with some modifications (Rödiger et al., 2010). Leaves of 4-week-old were collected and then exact $5 \mathrm{~g}$ of each sample were ground in $15 \mathrm{~mL}$ of isolation buffer, containing $1 \mathrm{mM}$ DL-dithiothreitol, $1 \%(\mathrm{w} / \mathrm{v})$ polyvinylpyrrolidone- 40 and $1 \%(\mathrm{w} / \mathrm{v})$ defatted bovine serum albumin, adjusted to $\mathrm{pH} 7.5$ with $\mathrm{KOH}$. The extraction was

TABLE 1 | List of primers for qRT-PCR.

\begin{tabular}{|c|c|c|}
\hline Gene & Accession number & Primer pairs \\
\hline \multirow[t]{2}{*}{$A T P \beta-1$} & At5g08670 & 5'-ACGGACAAATGAATGAGCC-3' \\
\hline & & 5'-ACAGCAGACGGGATACGAC-3' \\
\hline \multirow[t]{2}{*}{ ATP $\beta-2$} & At5g08690 & 5'-TGTGGCTGAGTATTTCCGTGAT-3' \\
\hline & & 5'-GCAGGGACATAGATGGCTTG-3' \\
\hline \multirow[t]{2}{*}{ ATP $\beta-3$} & At5g08680 & 5'-GACAACATCTTCCGTTCAC-3' \\
\hline & & 5'-ATGGCTTGGACAGAGGTAAT-3' \\
\hline \multirow[t]{2}{*}{$A T P_{\varepsilon}$} & At1g51650 & 5'-ATGCGGCGGTTCCGTTCT-3' \\
\hline & & 5'-AGGTTTCTGGGGCTTCC-3' \\
\hline \multirow[t]{2}{*}{ SAG12 } & At5g45890 & 5'-ACTGGTITCAAAGGTGTCTCGGCAT-3' \\
\hline & & 5'-ACGCCCAACAACATCCGCAGC-3' \\
\hline \multirow[t]{2}{*}{ SAG13 } & At2g29350 & 5'-AGCGACAACATAAGGACGAAC-3' \\
\hline & & 5'-AGACAAAGAAATGCCACAAGC-3' \\
\hline \multirow[t]{2}{*}{ SAG29 } & At5g13170 & 5'-TCGGCATCTTAGGAAACG-3' \\
\hline & & 5'-CGGTAGCGACTGGAAACT-3' \\
\hline \multirow[t]{2}{*}{ UBQ4 } & At5g20620 & 5'-GGGCACTCAAGTATCTTGTTAGC-3' \\
\hline & & 5'-TGCTGCCCAACATCAGGTT-3' \\
\hline
\end{tabular}

filtered through 2 layers of $25-\mu \mathrm{m}$ nylon mesh and centrifuged for $10 \mathrm{~min}$ at $3000 \mathrm{~g}$. The supernatant was transferred to a new tube and centrifuged for $15 \mathrm{~min}$ at $20000 \mathrm{~g}$, and the sediment was resuspended in $15 \mathrm{~mL}$ washing buffer before repeating the above steps. The resulting supernatant was discarded and the pellet was resuspended in $1 \mathrm{~mL}$ of washing buffer. The pellet's supernatant was carefully layered on top of a Percoll gradient and centrifuged for $60 \mathrm{~min}$ at $40000 \mathrm{~g}$. The mitochondrial fraction, which accumulated at the interphase between 27 and 50\% Percoll, was collected. The sediment was diluted with $1 \mathrm{~mL}$ of washing buffer and centrifuged for $15 \mathrm{~min}$ at $20000 \mathrm{~g}$. This was repeated three times. Finally, the pellet was resuspended in $1 \mathrm{~mL}$ of preservation buffer and stored. All steps involved in isolating mitochondria were conducted at $4^{\circ} \mathrm{C}$. Janus green $\mathrm{B}$ which has been considered specific for mitochondria was used to promise the isolated mitochondria were viable (Supplementary Figure S3) (Lyons et al., 1964).

\section{Measurement of Mitochondrial Activity and Mitochondrial Swelling}

The mitochondrial activity was determined according to previously described methods with some modifications (Du et al., 2014). Take $100 \mu \mathrm{L}$ of freshly prepared mitochondrial suspension sample stored at $4^{\circ} \mathrm{C}$ (with $100 \mu \mathrm{L}$ suspension buffer as blank control), add $40 \mu \mathrm{L}$ of $5 \mathrm{mg} \cdot \mathrm{mL}^{-1}$ MTT (3-(4,5-Dimethylthiazol2-yl)-2,5-Diphenyltetrazolium Bromide), incubate at $30^{\circ} \mathrm{C}$ for $30 \mathrm{~min}$, then add $100 \mu \mathrm{L}$ of isopropanol at room temperature for $20 \mathrm{~min}$, and the absorbance value of $\mathrm{A}_{570}$ was measured using a multi-function microplate reader, and the value thereof reflected mitochondrial activity.

Freshly prepared mitochondria were kept at $4^{\circ} \mathrm{C}$ prior to the reaction. The mitochondria removed rapidly in to the medium (25 mM sucrose, $0.5 \mathrm{mM} \mathrm{KH}_{2} \mathrm{PO}_{4}, 1 \mathrm{mM}$ Sodium succinate, and $\mathrm{pH}$ 7.2). Mitochondrial swelling was determined in all groups by measuring the change in the absorbance of the mitochondrial suspension at $540 \mathrm{~nm}$. The reaction conditions were set at $25^{\circ} \mathrm{C}$. According to this method, the more serious the mitochondrial swelling is, the smaller absorbance value at $540 \mathrm{~nm}$ becomes ( $\mathrm{Du}$ et al., 2014).

\section{Measurement of ATPase Activity and ATP Content in Mitochondria}

ATPase activity was assayed by measuring inorganic phosphate (Pi) from ATP, and Pi was determined according to Ames (Toribarn et al., 1966). In brief, ATPase activity was measured in $0.5 \mathrm{~mL}$ of reaction solution contained $0.25 \mathrm{~mL}$ mitochondrial suspension, $3 \mathrm{mM}$ ATP, $50 \mathrm{mM} \mathrm{KCl}, 1.5 \mathrm{mM} \mathrm{MgCl}_{2}, 50 \mathrm{mM}$ $\mathrm{NaCl}$ and $33 \mathrm{mM}$ Tris titrated to $\mathrm{pH} 6.5$ with HCl. Corrections were made using blanks without ATP. After the incubation of $30 \mathrm{~min}$ at $38^{\circ} \mathrm{C}, 100 \mu \mathrm{L} 20 \%(\mathrm{w} / \mathrm{v})$ trichloroacetic acid quenched the reaction. The liberated Pi was measured with a spectrophotometer at $700 \mathrm{~nm}$. ATPase activity was expressed in $\mu \mathrm{mol}$ Pi per mg protein per min, and the protein content was determined by the method (Lowry et al., 1951).

The amount of ATP in mitochondria was measured using the luciferin-luciferase assay. The assay was analyzed with an 
ENLITEN ATP assay bioluminescence detection kit, according to the manufacturer's recommended protocol. In addition, operational steps also based on previously published methods were performed with minor modification (Goh et al., 2004). The luminescence was integrated for $10 \mathrm{~s}$ using a multifunctional microplate reader. The actual ATP levels were calculated from an ATP standard curve, which was constructed using different concentrations of commercially supplied ATP.

\section{Measurement of the Cys Content in Mitochondria}

The L-Cys contents of mitochondria were quantitated using a method described previously (Fu et al., 2012). Briefly, $100 \mu \mathrm{L}$ samples were prepared from mitochondrial suspensions. L-Cys reacts with glacial acetic acid and ninhydrin reagent specifically, which has a maximum absorbance at $560 \mathrm{~nm}$.

\section{Measurement of the $\mathrm{H}_{2} \mathrm{~S}$ Production Rate and Content in Mitochondria}

Fresh mitochondrial suspensions were prepared to determine the production rate of $\mathrm{H}_{2} \mathrm{~S}$ from L-Cys and D-Cys. The assay was performed in accordance with methods described previously (Fu et al., 2012).

The endogenous $\mathrm{H}_{2} \mathrm{~S}$ contents of mitochondria were determined following the protocol described previously (Jin et al., 2017). Freshly prepared mitochondrial suspensions were homogenized with $1.5 \mathrm{~mL}$ of extraction buffer. The samples were then analyzed using a Four-channel Free Radical Analyzer with tissue electrodes. Different concentrations of NaHS (donor of $\mathrm{H}_{2} \mathrm{~S}$ ) were used against the available standard $\mathrm{H}_{2} \mathrm{~S}$ measurement curve.

\section{Statistical Analyses}

Each experiment was performed in triplicate, each with at least three biological replicates. All data were presented as the means \pm SEs. The results were statistically analyzed using a one-way analysis of variance with SPSS 16.0 software, and error bars were determined based on Tukey's multiple range test. $P$-value $<0.05$ was considered statistically significant.

\section{RESULTS}

\section{The $\mathrm{H}_{2} \mathrm{~S}$ Content Is Correlated With Leaf Senescence During Drought Stress}

The $\mathrm{H}_{2} \mathrm{~S}$ contents in leaves from different plants were quantified. Compared with WT, the $\mathrm{H}_{2} \mathrm{~S}$ content of the des 1 mutant decreased significantly and that of OE-DES1 increased significantly (Jin et al., 2017). The effects of $\mathrm{H}_{2} \mathrm{~S}$ on seedling growth under normal and drought-stress conditions were observed. Under normal conditions, the development of des 1 was much slower than WT and OE-DES1. Most leaves of des 1 showed wilting and turned yellow after 5 days under droughtstress conditions, while leaves of WT curled severely and those of $O E-D E S 1$ were green and expanded (Figure 1).

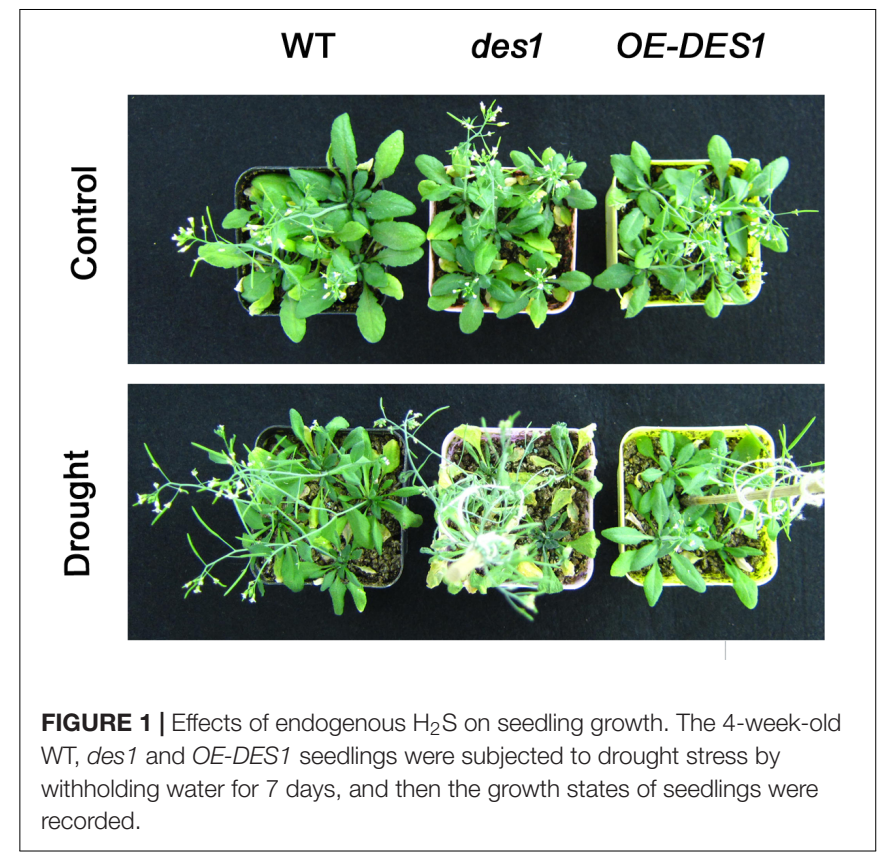

\section{Endogenous $\mathrm{H}_{2} \mathrm{~S}$ Affects the Transcriptional Levels of SAGs and ATP Synthase-Related Genes}

The gene expression levels of $A T P \beta-1, A T P \beta-2, A T P \beta-3, A T P \varepsilon$, $S A G 12, S A G 13$, and $S A G 29$ at the different growth stages of WT plants were analyzed using qRT-PCR with mRNA from the leaves of 2-, 4-, 6-, and 8-week-old plants. Compared with the expression at 2 weeks, the levels of $A T P \beta-1 \sim 3$ and $A T P \varepsilon$ were stimulated by 5-, 7.25-, 2.8-, and 1.6-fold at 4 weeks, respectively, and then dropped at 6 and 8 weeks (Figure 2A). During this period, the expression levels of SAG12, 13 and 29 gradually increased. SAG12 was the greatest responder, with expression up-regulated by 3.58 -fold at 6 weeks and 7.67 -fold at 8 weeks compared with the levels at 2 weeks (Figure 2B).

To study the effects of drought stress on the expression levels of these genes, qRT-PCR was performed using mRNA from the leaves of plants grown under two different conditions. Under normal conditions, the expression levels of ATP $\beta$ s increased significantly in des1 and OE-DES1 compared with WT, while $A T P \varepsilon$ decreased significantly (Figure 2C). The expression level of SAG12 was not different between des1 and WT, but decreased significantly in OE-DES1. Additionally, SAG13 and SAG29 expression levels were significantly downregulated in des1 and OE-DES1 compared with WT (Figure 2D). Under drought-stress conditions, the expression levels of ATP $\beta$ s were significantly lower in des 1 and OE-DES1, and that of ATPE was greater in des 1 compared with WT (Figure 2E). At the same time, compared with WT, SAG12 expression reduced in des 1 but significantly enhanced in OE-DES1, while SAG13 had the opposite trend. SAG29 expression was significantly down-regulated in both des 1 and OE-DES1 (Figure 2F). 
A

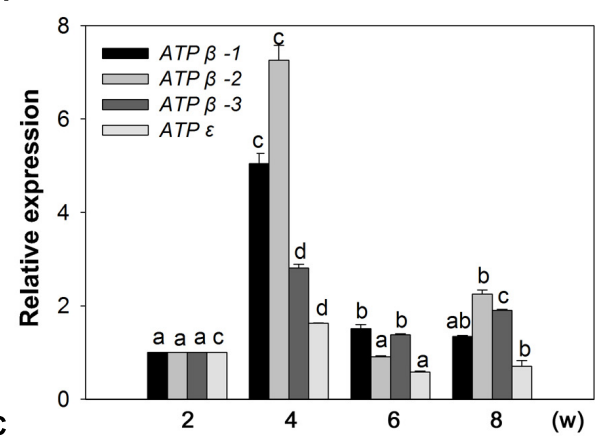

B

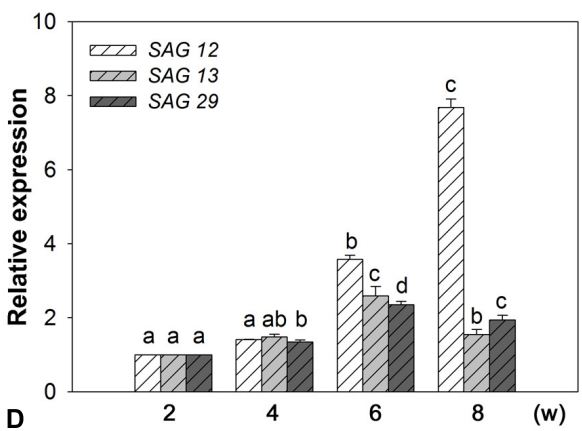

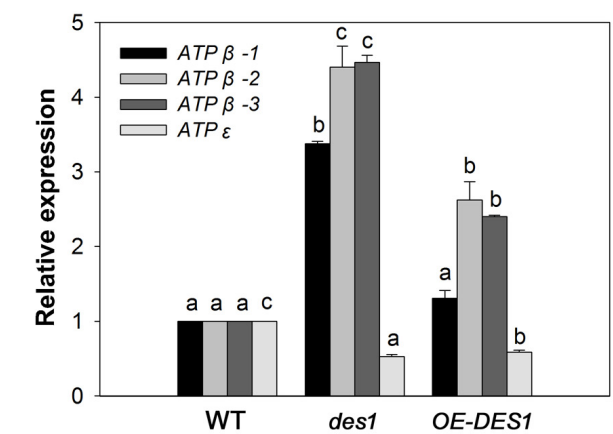

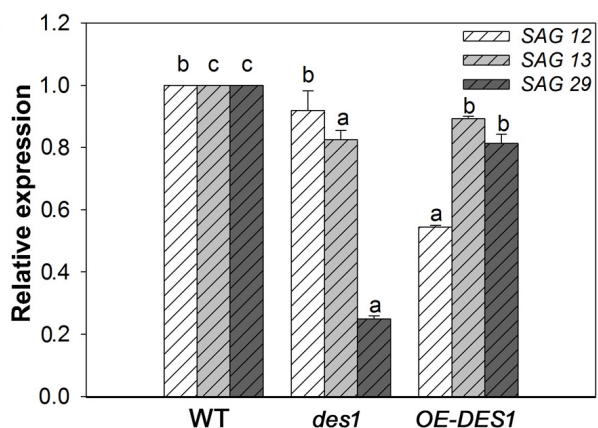

E

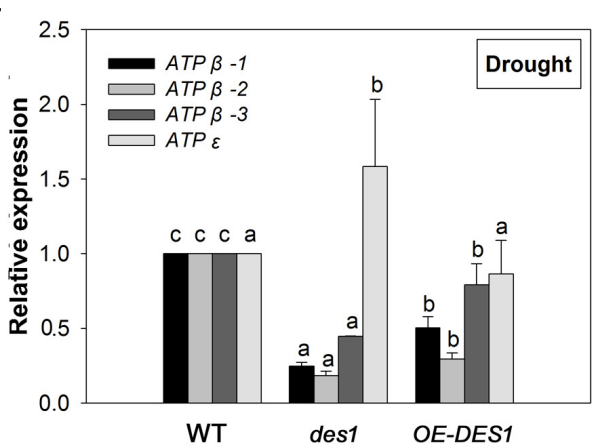

$\mathbf{F}$

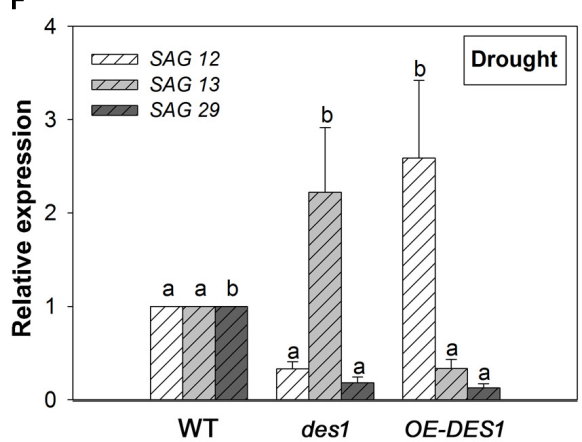

FIGURE 2 | Expression patterns of SAGs and ATP synthase-related genes. (A) Expression patterns of ATP synthase-related genes at different developmental stages. (B) Expression patterns of SAGs at different developmental stages. (C) Effects of endogenous $\mathrm{H}_{2} \mathrm{~S}$ on the expression levels of ATP synthase-related genes. (D) Effects of endogenous $\mathrm{H}_{2} \mathrm{~S}$ on the expression levels of SAGs. (E) Expression levels of ATP synthase-related genes under drought-stress conditions in WT, des 1 and OE-DES1. (F) Expression levels of SAGs under drought-stress conditions in WT, des1 and OE-DES1. Data are means \pm SEs of three independent experiments; bars marked with the same letter did not differ significantly at $p<0.05$.

\section{Endogenous $\mathrm{H}_{2} \mathrm{~S}$ Protected the Mitochondrial Ultrastructure in Leaves Under Drought-Stress Conditions}

Leaves of plants at different developmental stages were collected after withholding water for 1 week, and the ultrastructure of the mitochondria were observed using TEM. The representative ultrastructures at lower magnification are shown as Supplementary Figure S5. In 2-week-old young rosette tissues, the mitochondria had intact membranes, elaborate cristae and homogeneous matrixes were seen in all of the materials (Figures 3A-C). With seedling development, the mitochondrial membrane became deformed and the cristae swelled gradually in WT and des1, while OE-DES1 seedlings displayed relatively normal and numerous mitochondria (Figures 3D-I). In the aging leaves under drought stress for 1 week, the mitochondria of des 1 lost their internal structures and swollen cristae, which had internal contents that degraded and membrane that became incomplete. WT samples followed a similar change and the mitochondrial cristae suffered serious damage and tended to bubble. The mitochondrial structures of OE-DES1 were intact, with mitochondrial cristae having a tubular shape and no obvious swelling (Figures 3J-L).

\section{Endogenous $\mathrm{H}_{2} \mathrm{~S}$ Affected Mitochondrial Activity, Mitochondrial Swelling, ATPase Activity and ATP Content}

To identify the effects of $\mathrm{H}_{2} \mathrm{~S}$ on the physiological functions of mitochondria, the mitochondrial fractions in WT, des 1 


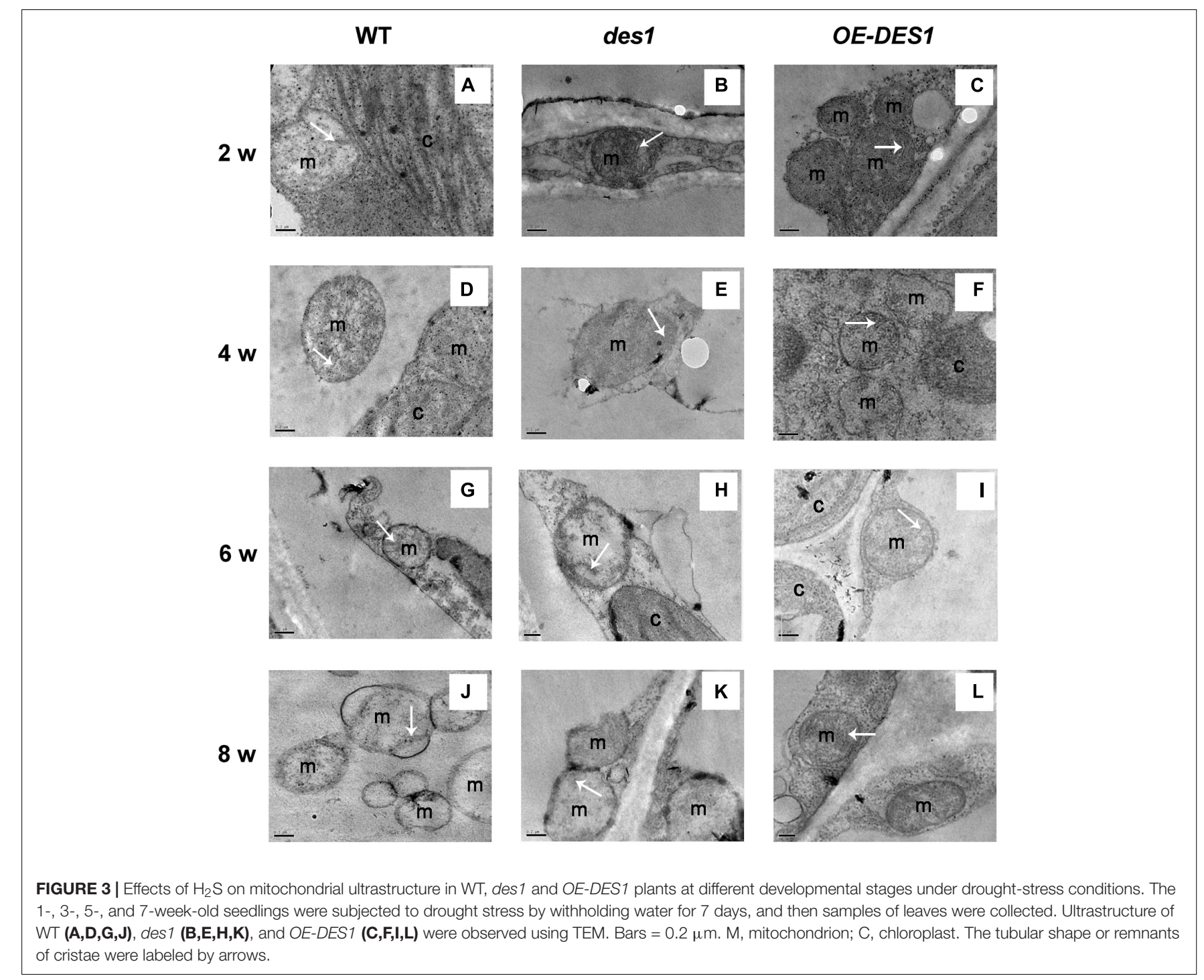

and OE-DES1 were independently isolated. The viability of mitochondria was detected with Janus Green B (Supplementary Figure S3). To control the purity of our mitochondrial fractions, the expressions of the marker protein in chloroplast and mitochondrion were determined, respectively (Supplementary Figure S4). Under drought-stress treatments, the mitochondrial activity was assessed using an MTT colorimetric assay at $\mathrm{OD}_{570}$. No significant differences were found among WT, des 1 and OE-DES1 (Figure 4A). The swelling of mitochondria is closely related to their activity levels. Consistent with the ultrastructure results, the swelling of mitochondria significantly increased in des 1 and significantly decreased in OE-DES1 compared with WT (Figure 4B). To further investigate the function of $\mathrm{H}_{2} \mathrm{~S}$ on energy production in mitochondria, the ATPase activity levels and ATP contents of mitochondria were detected. Under drought-stress conditions, ATPase activity levels of mitochondria decreased significantly in des1 and increased significantly in OE-DES1 compared with the WT (Figure 4C). In contrast, an opposite trend occurred for the ATP contents of different mitochondria, which indicated that des 1 has significantly more ATP and OE-DES1 has significantly less in comparison with WT (Figure 4D).

\section{Relationships Among the Cys Content, $\mathrm{H}_{2} \mathrm{~S}$ Content and $\mathrm{H}_{2} \mathrm{~S}$ Production Rate in Mitochondria}

To further confirm the regulatory role of $\mathrm{H}_{2} \mathrm{~S}$ on ATP production in mitochondria, we isolated the mitochondrial fractions and detected the contents of Cys and $\mathrm{H}_{2} \mathrm{~S}$, along with the $\mathrm{H}_{2} \mathrm{~S}$ production rate, in different cysteine substrates. Drought stress induced a significant increase in the Cys content of des1 compared with WT, and there was no significant difference in the content between OE-DES1 and WT (Figure 5A). The $\mathrm{H}_{2} \mathrm{~S}$ content revealed opposite results (Figure 5B). The enzyme-catalyzed degradation of Cys is considered the main pathway of $\mathrm{H}_{2} \mathrm{~S}$ production in plants. As expected, regardless of whether there was the L-Cys or D-Cys substrate, the 

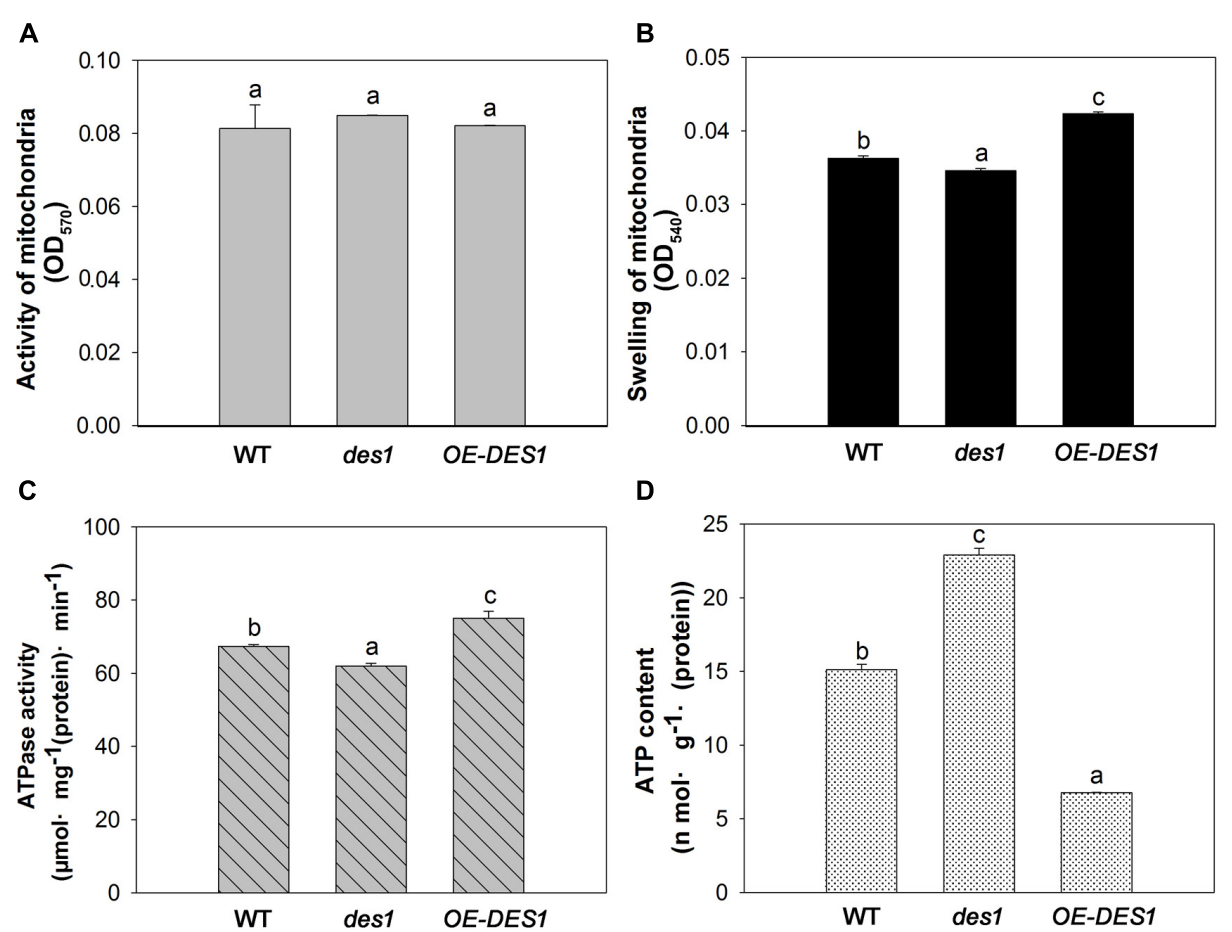

FIGURE 4 | Effects of $\mathrm{H}_{2} \mathrm{~S}$ on mitochondrial functions in WT, des1 and OE-DES1 under drought-stress conditions. Leaves of 4-week-old after withholding water for 1 week were collected and the mitochondria were isolated and purified. (A) Activity levels of mitochondria and (B) swelling of mitochondria according to the method previously described (Du et al., 2014). (C) ATPase activity levels of mitochondria according to the method previously described (Toribarn et al., 1966). (D) ATP contents of mitochondria in WT, des 1 and OE-DES1 were measured by the luciferin-luciferase (Promega, Madison, WI, United States). Data are means \pm SEs of three independent experiments; bars marked with the same letter did not differ significantly at $p<0.05$.

production rate of $\mathrm{H}_{2} \mathrm{~S}$ increased significantly in $O E-D E S 1$ and decreased significantly in des 1 , producing comparable yields (Figures 5C,D).

\section{DISCUSSION}

Leaf senescence represents a key developmental phase in the lives of both annual and perennial plants, and is as ordered and complex as any other developmental phase (BuchananWollaston, 1997). A large range of cDNA clones, representing genes, have been isolated and showed increased expression levels in senescing leaves. In our studies, the elevated expression of SAG12 is closely correlated with age-dependent senescence (Figure 2B), which was in agreement with a previous report (Kim et al., 2008), and revealed that senescence began with 6week-old leaves. Furthermore, the expression level of SAG12 decreased significantly in OE-DES1 under normal growth conditions compared with in WT (Figure 2D). In contrast, expression of SAG13 gradually increased with development and was significantly greater in des 1 than in WT and OEDES1 under drought-stress conditions (Figure 2F), which was specifically induced by environmental factors (Meyer et al., 2003). SAG29 is expressed primarily in senescing plant tissues and serves as a molecular link that integrates environmental stress responses into the senescence process (Chen et al., 2014). As shown here, the expression of SAG29 was significantly greater in $O E-D E S 1$ than in des1 under normal growth conditions (Figure 2D) and was not significantly different between des1 and $O E-D E S 1$ under drought-stress conditions (Figure 2F). Therefore, endogenous $\mathrm{H}_{2} \mathrm{~S}$ may regulate leaf senescence by participating in $S A G s^{\prime}$ expression under normal and droughtstress conditions.

Leaf senescence involves a genetically programmed loss of metabolic function associated with programmed cell death as the terminal phase. Mitochondria, which have elaborate cristae in young and mature Arabidopsis rosette tissues, only lose their internal structure, with swollen cristae, at the final programmed cell death stage of senescence when most of the cellular proteins and other reserves have been degraded and exported (Noctor et al., 2007). Thus, we observed the ultrastructures of mitochondria in leaves having unequal $\mathrm{H}_{2} \mathrm{~S}$ contents. Although the cristae in all cells swelled gradually during development, differences among the three plant materials from the same period were apparent. Mitochondrial damage was slightly mitigated in OE-DES1 compared with in the WT and des1 under drought-stress conditions (Figure 3). The absorbance at $\mathrm{OD}_{540}$, representing swelling of mitochondria, corroborated the previous result (Figure 4B). In consequence, physiological $\mathrm{H}_{2} \mathrm{~S}$ may protect the mitochondrial structures from injury induced by drought stress during plant senescence, which is consistent with previous studies in animals (Aslami et al., 2013; Yang et al., 2013; Du et al., 2014). 

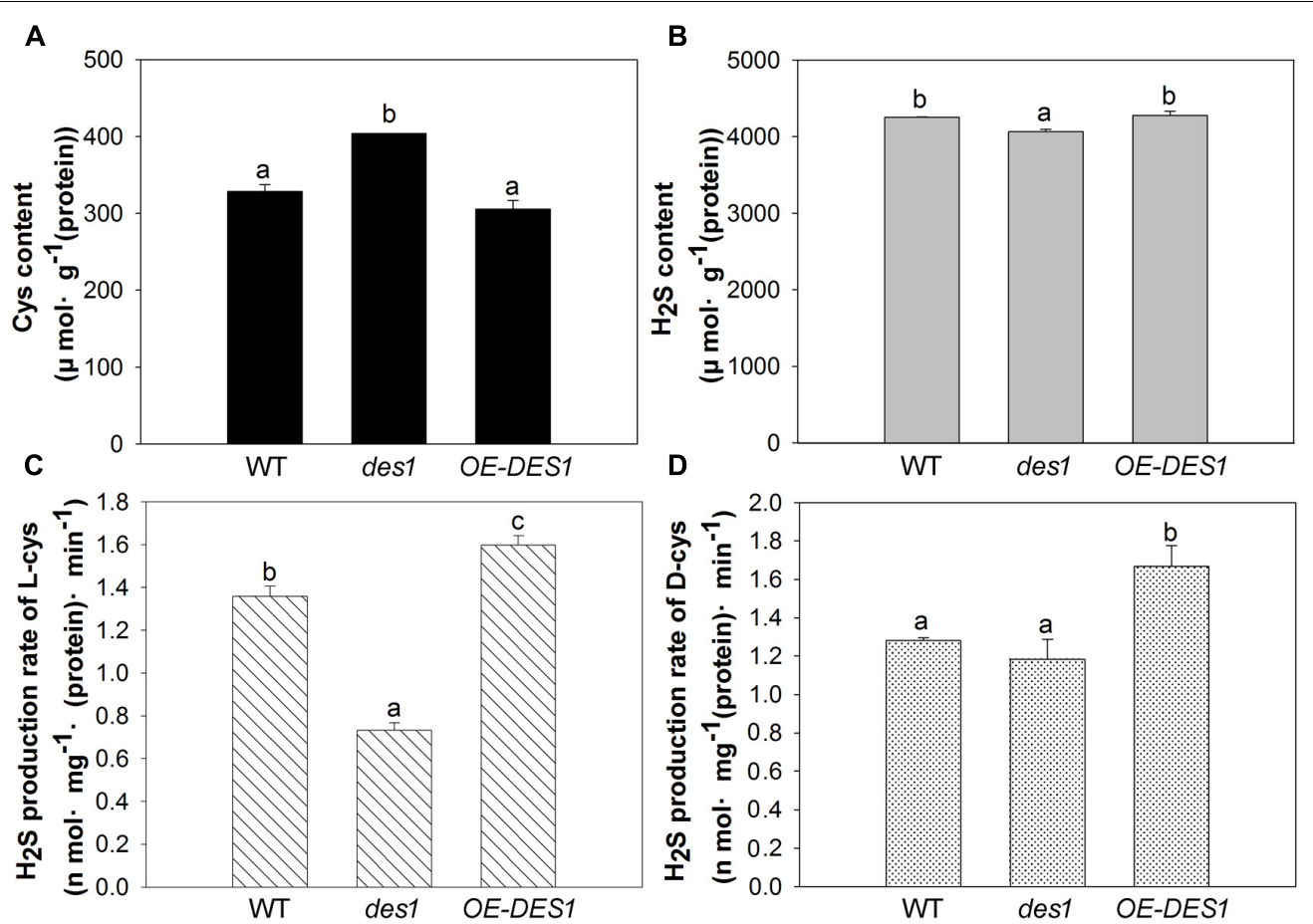

FIGURE 5 | Effects of drought stress on the $\mathrm{H}_{2}$ S-related physiological indices in the mitochondria of WT, des1 and OE-DES1. Leaves of 4-week-old after withholding water for 1 week were collected and the mitochondria were isolated and purified. (A) Cys contents of mitochondria were quantitated by the method described previously (Fu et al., 2012). (B) $\mathrm{H}_{2} \mathrm{~S}$ contents of mitochondria were measured with electrodes (TBR4100 WPI, Sarasota, FL, United States). (C) $\mathrm{H}_{2} \mathrm{~S}$ production rates of L-Cys and (D) $\mathrm{H}_{2} \mathrm{~S}$ production rates of D-Cys in mitochondria of WT, des1 and OE-DES1 were measured with methylene blue method (Fu et al., 2012). Data are means \pm SEs of three independent experiments; bars marked with the same letter did not differ significantly at $p<0.05$.

The three $\beta$ subunits, having an alternate arrangement in the ATPase complex, catalyze the synthesis of ATP from ADP and $\mathrm{Pi}$, and the $\varepsilon$ subunit is an endogenous inhibitor of the ATPase activity that may regulate ATPase activity (Kadoya et al., 2011). In the present study, the expression levels of $A T P \beta$ s and $A T P \varepsilon$ were significantly up-regulated by 4 weeks and then declined (Figure 2A), which might indicate that the demand for ATPase was greater at the seeding stage but lower during leaf senescence. ATP $\beta$ s' expression levels significantly increased in des 1 under normal conditions but decreased under droughtstress conditions compared with OE-DES1, while the expression of $A T P \varepsilon$ showed the opposite trend during the same time (Figures 2C,E). Under drought-stress treatment, the ATPase activities of mitochondria markedly increased in OE-DES1 and decreased in des 1 compared with WT (Figure 4C). The ATPase activity was in accordance with the expression levels of ATPase genes (Figures 2E, 4C), suggesting that $\mathrm{H}_{2} \mathrm{~S}$ enhanced the activity of ATPase by upregulating the expression levels of $A T P \beta$ s and downregulating the expression level of $A T P \varepsilon$ in response to leaf senescence induced by drought stress.

High ATP synthase activity means high ATP production. However, in contrast to the ATPase activity, the ATP concentration was the greatest in des 1 and the lowest in OE-DES1 compared with WT (Figures 4C,D). In other words, there is a lower ATPase activity which may corroborate with an accumulation of ATP in des1, and vice versa in OE-DES1.
In fact, the ATP content we measured shows the sum of positive ATP generation and negative ATP consumption. That is, ATP consumption might be higher in OE-DES1 which has lower ATP content. In addition, it is also plausible that the lower $\mathrm{H}_{2} \mathrm{~S}$ content measured in des 1 impacts the ATP production under drought-stress conditions. It has previously been reported in mammals that $\mathrm{H}_{2} \mathrm{~S}$ decreases the ATP content of mitochondria in smooth muscle cells under hypoxia-stress conditions and in brain cells under normoxic conditions (Fu et al., 2012). Individuals with high metabolic capacities may possess adaptations against aging (Niitepõld and Hanski, 2013). Thus, we hypothesized that $\mathrm{H}_{2} \mathrm{~S}$ might preserve ATPase activities to meet the needs of the higher metabolism in OE-DES1, which had leaves that were relatively younger during drought stress (Figure 1).

DES1, localized in the cytoplasm, is the main enzyme producing $\mathrm{H}_{2} \mathrm{~S}$ in plants. So, it is very interesting that DES1 mutation affected the $\mathrm{H}_{2} \mathrm{~S}$ production in mitochondrion. Why? It has been reported that cystathionine $\gamma$-lyase (the main enzyme of $\mathrm{H}_{2} \mathrm{~S}$ production in animal cells, also localized in cytoplasm) can translocate to mitochondria on hypoxic stress stimulation. Subsequently $\mathrm{H}_{2} \mathrm{~S}$ production inside mitochondria was promoted, and mitochondrial ATP production was sustained (Fu et al., 2012). As eukaryotes, there are many similarities in their physiological and biochemical processes in both animals and plants. Especially in the field of gasotransmitter research, 


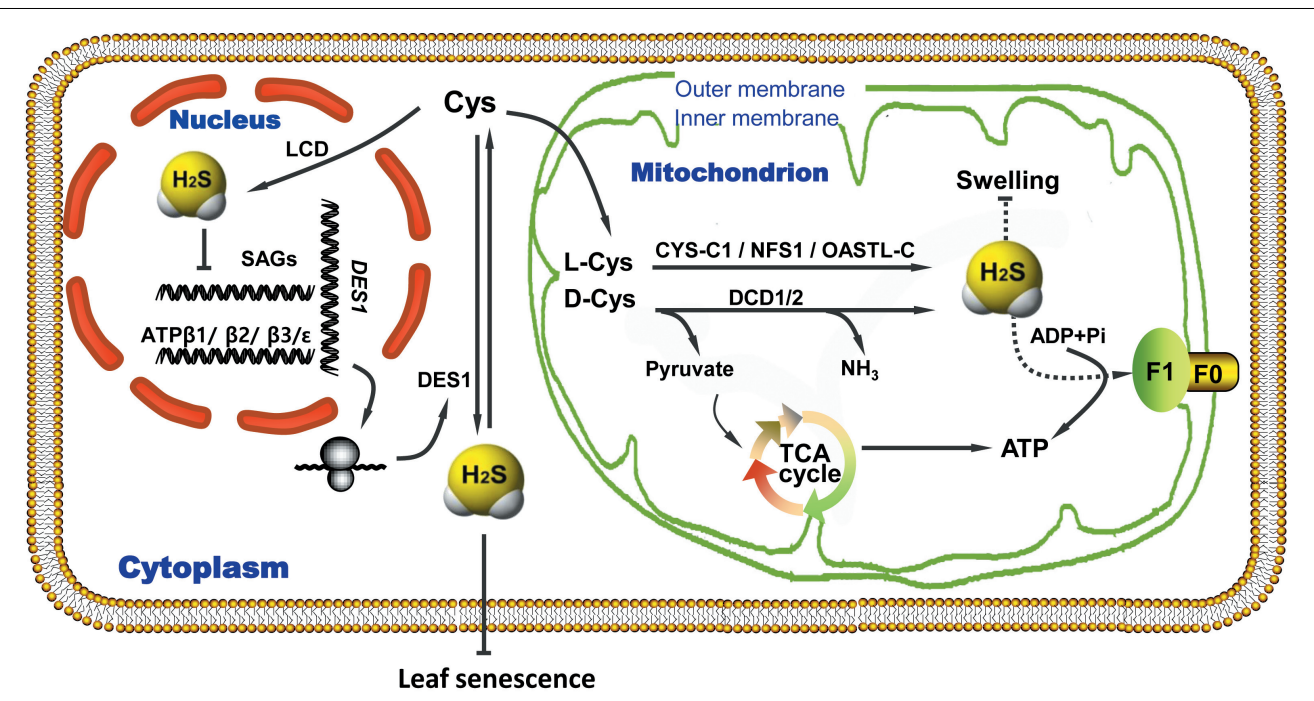

FIGURE 6 | Proposed regulatory model of ATP production induced by endogenous $\mathrm{H}_{2} \mathrm{~S}$ to delay leaf senescence under drought-stress conditions. ADP, adenosine diphosphate; ATP, adenosine tiphosphate; Cys, cysteine; CYS, cyanoalanine synthase; DES1, cysteine desulfhydrase; $F_{0} / F_{1}$, complex of ATP synthase; $H_{2} S$, hydrogen sulfide; LCD/DCD, L/D-cysteine desulfhydrase; NFS1, cysteine desulfurase; OASTL, O-acetyl-L-serine(thiol)lyase; SAGs, senescence-associated genes; TCA, tricarboxylic acid; Arrow end, activation; Blunt end, inactivation.

numerous studies have confirmed that the analogy from animal to plant was correct, which provided us with a valuable research idea. It is quite possible that DES1 affects the production of $\mathrm{H}_{2} \mathrm{~S}$ in mitochondria and subsequently affects energy production in mitochondria through the similar mechanism of action. It should be actually investigated more deeply in the future.

$\mathrm{H}_{2} \mathrm{~S}$ metabolism occurs in mitochondria, and $\mathrm{H}_{2} \mathrm{~S}$ improves mitochondrial ATP production in mammalian cells under stress conditions (Fu et al., 2012). The des 1 mutant of Arabidopsis displayed premature aging under Cd stress (Álvarez et al., 2010), and $\mathrm{H}_{2} \mathrm{~S}$-induced stomatal closure requires $\mathrm{ABC}$ transporter-dependent extracellular ATP production (Wang et al., 2015). In this present study, we explored the regulation of energy produced by endogenous $\mathrm{H}_{2} \mathrm{~S}$ in plants. The $\mathrm{H}_{2} \mathrm{~S}$ content and $\mathrm{H}_{2} \mathrm{~S}$ production rate based on L-Cys and $\mathrm{D}$-Cys were enhanced in the mitochondria of OE-DES1, indicating that the above results are effects of $\mathrm{H}_{2} \mathrm{~S}$ in mitochondria (Figure 5). In fact, several enzymes related to $\mathrm{H}_{2} \mathrm{~S}$ production are localized on the mitochondria, including DCys desulphydrase1 (DCD1, At1g48420), DCD2 (At3g26115), cysteine desulfurase (NFS1, At5g65720), cyanoalanine synthase (CYS-C1, At3g61440) and O-acetyl-L-serine(thiol)lyase (OASTLC, At3g59760). We compared their expression levels in WT between normal and drought-stress conditions. They all responded to drought stress. The expression levels of $D C D 2$, CYS-C1, and OASTL-C were significantly increased compared with WT (Supplementary Figure S6), which implies these three proteins may play roles in the regulation of mitochondrial energy metabolism during leaf senescence. Subsequent studies with mutants of these enzymes should clarify the $\mathrm{H}_{2} \mathrm{~S}$ associated regulatory mechanism of mitochondrial energy metabolism.

\section{CONCLUSION}

Endogenous $\mathrm{H}_{2} \mathrm{~S}$ mitigates mitochondrial swelling, preserves energy production and protects cellular metabolism during leaf senescence induced by drought stress (Figure 6). As such, $\mathrm{H}_{2} \mathrm{~S}$ delays leaf senescence by acting as a regulator of energy production in mitochondria. These findings increase our understanding of the physiological functions of $\mathrm{H}_{2} \mathrm{~S}$ and the regulation of energy metabolism in plants.

\section{AUTHOR CONTRIBUTIONS}

GY and YP designed the original research project. LS and ZJ performed all the experiments. LS and ZJ analyzed the data and wrote the manuscript. GY and YP revised the writing. All authors have read and approved the manuscript.

\section{FUNDING}

This work was funded by grants from the National Natural Science Foundation of China (Grant Nos. 31672140 to ZJ and 31671605 to YP) and University Science and Technology Innovation Project of Shanxi (Grant No. 2016110 to ZJ).

\section{SUPPLEMENTARY MATERIAL}

The Supplementary Material for this article can be found online at: https://www.frontiersin.org/articles/10.3389/fpls.2018.01722/ full\#supplementary-material 


\section{REFERENCES}

Álvarez, C., Calo, L., Romero, L. C., Garcıá, I., and Gotor, C. (2010). An O-acetylserine(thiol)lyase homolog with L-cysteine desulfhydrase activity regulates cysteine homeostasis in Arabidopsis. Plant Physiol. 152, 656-669. doi: 10.1104/pp.109.147975

Álvarez, C., García, I., Moreno, I., Pérez-Pérez, M. E., Crespo, J. L., Romero, L. C., et al. (2012). Cysteine-generated sulfide in the cytosol negatively regulates autophagy and modulates the transcriptional profile in Arabidopsis. Plant Cell 24, 4621-4634. doi: 10.1105/tpc.112.105403

Aslami, H., Pulskens, W. P., Kuipers, M. T., Bos, A. P., Kuilenburg, A. B. P., Wanders, R. J. A., et al. (2013). Hydrogen sulfide donor NaHS reduces organ injury in a rat model of pneumococcal pneumosepsis, associated with improved bio-energetic status. PLoS One 8:e63497. doi: 10.1371/journal.pone.0063497

Buchanan-Wollaston, V. (1997). The molecular biology of leaf senescence. J. Exp. Bot. 48, 181-199. doi: 10.1093/jxb/48.2.181

Chen, J., Wu, F. H., Wang, W. H., Zheng, C. J., Lin, G. H., Dong, X. J., et al. (2011). Hydrogen sulphide enhances photosynthesis through promoting chloroplast biogenesis, photosynthetic enzyme expression, and thiol redox modification in Spinacia oleracea seedlings. J. Exp. Bot. 62, 4481-4493. doi: 10.1093/jxb/err145

Chen, M., Maodzeka, A., Zhou, L., Ali, E., Wang, Z., and Jiang, L. X. (2014). Removal of DELLA repression promotes leaf senescence in Arabidopsis. Plant Sci. 21, 26-34. doi: 10.1016/j.plantsci.2013.11.016

Dooley, F. D., Nair, S. P., and Ward, P. D. (2013). Increased growth and germination success in plants following hydrogen sulfide administration. PLoS One 8:e62048. doi: 10.1371/journal.pone.0062048

Du, Q., Wang, C., Zhang, N., Li, G. F., Zhang, M., Li, L. P., et al. (2014). In vivo study of the effects of exogenous hydrogen sulfide on lung mitochondria in acute lung injury in rats. BMC Anesthesiol. 14:117. doi: 10.1186/1471-225314-117

Du, X. Z., Jin, Z. P., Liu, D. M., Yang, G. D., and Pei, Y. X. (2017). Hydrogen sulfide alleviates the cold stress through MPK4 in Arabidopsis thaliana. Plant Physiol. Biochem. 120, 112-119. doi: 10.1016/j.plaphy.2017.09.028

Fang, H. H., Liu, Z. Q., Jin, Z., Zhang, L. P., Liu, D. M., and Pei, Y. X. (2016). An emphasis of hydrogen sulfide-cysteine cycle on enhancing the tolerance to chromium stress in Arabidopsis. Environ. Pollut. 213, 870-877. doi: 10.1016/j. envpol.2016.03.035

Fang, H. H., Liu, Z. Q., Long, Y. P., Liang, Y. L., Jin, Z. P., Zhang, L. P., et al. (2017). The $\mathrm{Ca}^{2+} /$ calmodulin2-binding transcription factor TGA3 elevates $L C D$ expression and $\mathrm{H}_{2} \mathrm{~S}$ production to bolster $\mathrm{Cr}^{6+}$ tolerance in Arabidopsis. Plant J. 91, 1038-1050. doi: 10.1111/tpj.13627

Fu, M., Zhang, W. H., Wu, L. Y., Yang, G. D., Li, H. Z., and Wang, R. (2012). Hydrogen sulfide $\left(\mathrm{H}_{2} \mathrm{~S}\right)$ metabolism in mitochondria and its regulatory role in energy production. PNAS. 109, 2943-2948. doi: 10.1073/pnas.1115634109

Gao, S. P., Hu, K. D., Hu, L. Y., Li, Y. H., Han, Y., Wang, H. L., et al. (2013). Hydrogen sulfide delays postharvest senescence and plays an antioxidative role in fresh-cut Kiwifruit. Hortscience 48, 1385-1392.

Goh, C. H., Jung, K. H., Roberts, S. K., McAinsh, M. R., Hetherington, A. M., Park, Y., et al. (2004). Mitochondria provide the main source of cytosolic ATP for activation of outward-rectifying $\mathrm{K}^{+}$channels in mesophyll protoplast of chlorophyll-deficient mutant rice (OsCHLH) seedlings. J. Biol. Chem. 279, 6874-6882. doi: 10.1074/jbc.M309071200

Gotor, C., García, I., Crespo, J. L., and Romero, L. C. (2013). Sulfide as a signaling molecule in autophagy. Autophagy 9, 609-611. doi: 10.4161/auto.23460

Jin, Z. P., and Pei, Y. X. (2015). Physiological implications of hydrogen sulfide in plants: pleasant exploration behind its unpleasant odour. Oxid. Med. Cell Longev. 2015:397502. doi: 10.1155/2015/397502

Jin, Z. P., and Pei, Y. X. (2016). Hydrogen sulfide: the shutter button of stomata in plants. Sci. China Life Sci. 59, 1187-1188. doi: 10.1007/s11427-016-0265-3

Jin, Z. P., Shen, J. J., Qiao, Z. J., Yang, G. D., Wang, R., and Pei, Y. X. (2011). Hydrogen sulfide improves drought resistance in Arabidopsis thaliana. Biochem. Biophys. Res. Commun. 414, 481-486. doi: 10.1016/j.bbrc.2011.09.090

Jin, Z. P., Wang, Z. Q., Ma, Q. X., Sun, L. M., Zhang, L. P., Liu, Z. Q., et al. (2017). Hydrogen sulfide mediates ion fluxes inducing stomatal closure in response to drought stress in Arabidopsis thaliana. Plant Soil 419, 141-152. doi: $10.1007 /$ s11104-017-3335-5
Jin, Z. P., Xue, S. W., Luo, Y. N., Tian, B. H., Fang, H. H., Li, H., et al. (2013). Hydrogen sulfide interacting with abscisic acid in stomatal regulation responses to drought stress in Arabidopsis. Plant Physiol. Biochem. 62, 41-46. doi: 10. 1016/j.plaphy.2012.10.017

Kadoya, F., Kato, S., Watanabe, K., and Kato-Yamada, Y. (2011). ATP binding to the $\epsilon$ subunit of thermophilic ATP synthase is crucial for efficient coupling of ATPase and $\mathrm{H}^{+}$pump activities. Biochem. J. 437, 135-140. doi: 10.1042/ BJ20110443

Kato, Y., Matsui, T., Tanaka, N., Muneyuki, E., Hisabori, T., and Yoshida, M. (1997). Thermophilic $\mathrm{F}_{1}$-ATPase is activated without dissociation of an endogenous inhibitor, $\varepsilon$ subunit. J. Biol. Chem. 272, 24906-24912. doi: 10.1074/ jbc. 272.40 .24906

Kim, C. Y., Bove, J., and Assmann, S. M. (2008). Overexpression of woundresponsive RNA-binding proteins induces leaf senescence and hypersensitivelike cell death. New Phytol. 180, 57-70. doi: 10.1111/j.1469-8137.2008. 02557.x

Li, Z. G., Gong, M., Xie, H., Yang, L., and Li, J. (2012). Hydrogen sulfide donor sodium hydrosulfide-induced heat tolerance in tobacco (Nicotiana tabacum L.) suspension cultured cells and involvement of $\mathrm{Ca}^{2+}$ and calmodulin. Plant Sci. 18, 185-189. doi: 10.1016/j.plantsci.2011.10.006

Lowry, O. H., Rosebrough, N. J., Farr, A. L., and Randall, R. J. (1951). Protein measurement with the folin phenol reagent. J. Biol. Chem. 193, 265-275.

Lyons, J., Wheaton, T., and Pratt, H. (1964). Relationship between the physical nature of mitochondrial membranes and chilling sensitivity in plants. Plant Physiol. 39, 262-268. doi: 10.1104/pp.39.2.262

Meyer, T., Burow, M., Bauer, M., and Papenbrock, J. (2003). Arabidopsis sulfurtransferases: investigation of their function during senescence and in cyanide detoxification. Planta 217, 1-10. doi: 10.1007/s00425-0020964-5

Munné-Bosch, S., and Alegre, L. (2004). Leaf senescence contributes to plant survival under drought stress. Funct. Plant Biol. 31, 203-216. doi: 10.1071/ FP03236

Niitepõld, K., and Hanski, I. (2013). A long life in the fast lane: positive association between peak metabolic rate and lifespan in a butterfly. J. Exp. Bot. 216, 1388-1397. doi: 10.1242/jeb.080739

Noctor, G., Paepe, R. D., and Foyer, C. H. (2007). Mitochondrial redox biology and homeostasis in plants. Trends Plant Sci. 12, 125-134. doi: 10.1016/j.tplants.2007. 01.005

Papenbrock, J., Riemenschneider, A., Kamp, A., Schulz-Vogt, H. N., and Schmidt, A. (2007). Characterization of cysteine-degrading and $\mathrm{H}_{2}$ S-releasing enzymes of higher plants-from the field to the test tube and back. Plant Biol. 9, 582-588. doi: 10.1055/s-2007-965424

Rödiger, A., Baudisch, B., and Klösgen, R. B. (2010). Simultaneous isolation of intact mitochondria and chloroplasts from a single pulping of plant tissue. J. Plant Physiol. 167, 620-624. doi: 10.1016/j.jplph.2009.11.013

Rondelez, Y., Tresset, G., Nakashima, T., Kato-Yamada, Y., Fujita, H., Takeuchi, S., et al. (2005). Highly coupled ATP synthesis by $\mathrm{F}_{1}$-ATPase single molecules. Nature 433, 773-777. doi: 10.1038/nature03277

Sõti, C., Sreedhar, A. S., and Csermely, P. (2003). Apoptosis, necrosis and cellular senescence: chaperone occupancy as a potential switch. Aging Cell 2, 39-45. doi: 10.1046/j.1474-9728.2003.00031.x

Toribarn, J. R., Chen, P. S., and Warner, H. (1966). Assay of inorganic phosphate, total phosphate and phosphatases. Method Enzymol. 8, $115-118$.

Wang, L. X., Ma, X. Y., Che, Y. M., Hou, L. X., Liu, X., and Zhang, W. (2015). Extracellular ATP mediates $\mathrm{H}_{2} \mathrm{~S}$-regulated stomatal movements and guard cell $\mathrm{K}^{+}$current in a $\mathrm{H}_{2} \mathrm{O}_{2}$-dependent manner in Arabidopsis. Sci. Bull. 60, 419-427. doi: 10.1007/s11434-014-0659-x

Wang, R. (2012). Physiological implications of hydrogen sulfide: a whiff exploration that blossomed. Physiol. Rev. 92, 791-896. doi: 10.1152/physrev. 00017.2011

Wang, W. G., Yang, X. L., Silanes, I. L., Carling, D., and Gorospe, M. (2003). Increased AMP: ATP ratio and AMP-activated protein kinase activity during celluar senescence linked to reduced HuR function. J. Bio. Chem. 278, 2701627023. 
Yang, G. D., Zhao, K. X., Ju, Y. J., Mani, S., Cao, Q. H., Puukila, S., et al. (2013). Hydrogen sulfide protects against cellular senescence via S-sulfhydration of Keap1 and activation of Nrf2. Antioxid. Redox Sign. 18, 1906-1919. doi: 10. 1089/ars.2012.4645

Zhang, H., Hu, L. Y., Hu, K. D., He, Y. D., Wang, S. H., and Luo, J. P. (2008). Hydrogen sulfide promotes wheat seed germination and alleviates oxidative damage against copper stress. J. Integr. Plant Biol. 50, 1518-1529. doi: 10.1111/ j.1744-7909.2008.00769.x

Zhang, H., Hu, S. L., Zhang, Z. J., Hu, L. Y., Jiang, C. X., Wei, Z. J., et al. (2011). Hydrogen sulfide acts as a regulator of flower senescence in plants. Postharvest Biol. Technol. 60, 251-257. doi: 10.1016/j.postharvbio.2011.01.006

Zhang, H., Tang, J., Liu, X. P., Wang, Y., Yu, W., Peng, W. Y., et al. (2009). Hydrogen sulfide promotes root organogenesis in Ipomoea batatas, Salix matsudana and Glycine max. J. Integr. Plant Biol. 51, 1086-1094. doi: 10.1111/j. 1744-7909.2009.00885.x

Conflict of Interest Statement: The authors declare that the research was conducted in the absence of any commercial or financial relationships that could be construed as a potential conflict of interest.

Copyright (๑) 2018 Jin, Sun, Yang and Pei. This is an open-access article distributed under the terms of the Creative Commons Attribution License (CC BY). The use, distribution or reproduction in other forums is permitted, provided the original author(s) and the copyright owner(s) are credited and that the original publication in this journal is cited, in accordance with accepted academic practice. No use, distribution or reproduction is permitted which does not comply with these terms. 